\title{
Assessing the safety and feasibility of metastasis- directed radiation and neoadjuvant hormone and radiation therapy followed by radical prostatectomy for treating oligometastatic prostate cancer: protocol for an open-label, dose-escalation, single- centre phase I clinical trial
}

\section{Xianzhi Zhao}

Department of Radiation Oncology, Shanghai Changhai Hospital, Navy Medical University

\section{Yu-Tian Xiao}

Department of Urology, Shanghai Changhai Hospital, Navy Medical University

Yue Yang

Department of Urology, Shanghai Changhai Hospital, Navy Medical University

Yusheng Ye

Department of Radiation Oncology, Shanghai Changhai Hospital, Navy Medical University

Yifan Chang

Department of Urology, Shanghai Changhai Hospital, Navy Medical University

\section{Lingong Jiang}

Department of Radiation Oncology, Shanghai Changhai Hospital, Navy Medical University

\section{Md Eftekhar}

Department of Family Medicine, CanAm International Medical Center

\section{Shancheng Ren}

Department of Urology, Shanghai Changhai Hospital, Navy Medical University

Huojun Zhang ( $\nabla$ wang_juan_2015@163.com )

Changhai Hospital

\section{Study protocol}

Keywords: Oligometastatic prostate cancer, neoadjuvant radiation therapy, radical prostatectomy, SBRT, safety, feasibility

Posted Date: March 24th, 2020

DOl: https://doi.org/10.21203/rs.3.rs-18507/v1 
License: (c) (i) This work is licensed under a Creative Commons Attribution 4.0 International License. Read Full License 


\section{Abstract}

\section{Background}

The systemic therapy is currently recommended for patients with oligometastatic prostate cancer, however the prognosis has not been satisfactory. Therefore, it is necessary to explore more effective treatment to improve the prognosis. Oligometastatic prostate cancer is a special subgroup in patients with advanced cancer. The paragrim of treatment is shifting to a more aggressive approach. Stereotactic body radiotherapy (SBRT) is an emerging treatment alternative for patients with oligometastases with minimal toxic effects. What's more, accumulating studies have proved safety as well as feasibility of radical prostatectomy and local or metastasis-directed radiotherapy for oligometastatic patients. The aim of this phase I prospective trial is to demonstrate the early evidence of safety and feasibility of metastasis-directed radiation and neoadjuvant hormone and radiation therapy followed by radical prostatectomy for treating oligometastatic prostate cancer

\section{Methods}

The patients with oligometastatic prostate cancer received 1 month of neoadjuvant androgen deprivation therapy (ADT) followed by metastasis-directed radition and abdominal or pelvic radiotherapy. Then offer radical prostatectomy at the interval of 4-8 weeks after radiotherapy and adjuvant ADT for 2 years. The primary endpoints of the study are safety as assessed by CTCAE 5.0 grading scale and intraoperative and postoperative day-30 morbidity. Secondary endpoints include the positive rate of post-operative edge, biochemical progression-free survival (bPFS), postoperative continence and sexual function recovery, quality of life (QoL), overall survival (OS) and adverse reactions of ADT.

\section{Discussion}

This is the first, phase I trial assessing the safety and feasibility of metastasis-directed radiation and neoadjuvant hormone and radiation therapy followed by radical prostatectomy treatment for patients with oligometastatic prostate cancer. If positive, the results of this trial may help to design subsequent phase II trials exploring the role of metastasis-directed radiation and neoadjuvant hormone and radiation therapy followed by radical prostatectomy in the randomized controlled study.

\section{Background}

Prostate cancer $(\mathrm{PCa})$ is the most common malignancy in men with an incidence rate of $29.3 \%$. It is also the leading cause of death among cancer entities with a mortality rate of $7.6 \%$ globally [1]. Traditionally, radical prostatectomy is recommended as the standard therapy for localized prostate cancer. However, if the patients present with evidence of metastasis even if it is a single positive lymph node, aggressive treatments are precluded and systemic therapies such as androgen deprivation therapy (ADT) or chemotherapy are strongly recommended $[2,3]$. The current paradigm for treatment of advanced PCa is shifting towards more aggressive approach. The last two decades have witnessed a great progress in 
surgical and radiation techniques, such as robotic-assisted laparoscopic radical prostatectomy (RALP), intensity modulated radiation therapy (IMRT) and stereotactic body radiotherapy (SBRT). The emerging evidence indicate that the various aggressive approaches might provide survival benefits to patients with lymph node-positive and metastatic PCa [4-8].

Oligometastatic prostate cancer has been proposed as an intermediate stage of cancer spread between the localized disease and the widespread metastases. The curative treatment of oligometastatic prostate cancer actually requires a 3-step method: firstly, local consolidate therapy for the primary tumor; secondly, metastasis-directed therapy and; thirdly, systemic ADT or chemotherapy. With the development of DaVinci Robotic System, RALP has become more clinically significant for tumor control, functional outcomes and complication manipulation for locally advanced prostate cancer $[9,10]$. As an increasingly safe and effective treatment, the value of metastasis-directed radiation therapy remains promising for oligometastatic Pca [10]. In this study, we propose the use of radiation therapy for local as well as distant metastatic lesions thus changing the current status of oligometastasis into advanced prostate cancer for further treatment.

For patients with locally advanced prostate cancer, multimodality approach including radical prostatectomy with lymph node dissection and adjuvant long-term ADT plus radiotherapy is strongly recommended to minimize the recurrence risk [2, 3]. In addition, adverse pathological features from radical surgery can identify patients who are likely to benefit from adjuvant radiotherapy. The phase III randomized controlled trials have demonstrated that locally advanced PCa patients can benefit from adjuvant radiotherapy in terms of tumor control and biochemical progression [11-13]. Randomized clinical trials of other malignancies such as rectal cancer have demonstrated safety and efficacy of neoadjuvant radiotherapy with tolerable toxicities [14]. Compared to postoperative radiation, preoperative therapy has the following advantages. Firstly, there is improved oxygenation of target tissues in neoadjuvant setting that decreases the radio-resistance without altering the blood supply of prostate [14]. Secondly, the neoadjuvant radiation requires a lower dose to achieve the equivalent level of tumor control which helps in reducing potential side effects [15]. Thirdly, pre-operative radiation can decrease viable cancer cells at the time of radical prostatectomy and sterilize extra-prostatic clonogenic stem cells in close proximity to the gland leading to reduction in probability of local recurrence [16]. Furthermore, the metastasis-directed radiation can decrease the total tumor burden of oligometastatic PCa patients. Preoperative radiation partially increases down-staging, and incorporation with neoadjuvant ADT further decreases the tumor stage and also aims micro-metastasis. Furthermore, the duration of treatment becomes shorter when radiation is given preoperatively.

Therefore, randomized controlled trials are required to address the promising effects of metastasisdirected radiation and neoadjuvant hormone and radiation therapy plus radical prostatectomy for oligometastatic prostate cancer. Hereby, we present a phase I study in order to demonstrate early evidence of safety and efficacy of the intervention.

\section{Methods/design}


This study was approved by the Ethics committee of the Shanghai Changhai Hospital (CHEC2019-110) and is registered on ChiCTR (CHiCTR1900025743). This is a phase I, prospective, single-arm study.

The main objective of the phase I trial is to determine whether metastasis-directed radiation and neoadjuvant hormone and radiation therapy followed by radical prostatectomy for the treatment of oligometastatic prostate cancer is safe and well tolerated, by assessing the occurrence of radiotherapyrelated complications along with intra operative and postoperative day-30 morbidity.

\section{Objectives}

Primary endpoint The primary endpoints of the study were safety of radiotherapy as assessed by CTCAE 5.0 grading scale along with intraoperative and postoperative day-30 morbidity. The radiotherapy-related complications encompassed the genitourinary (GU) toxicity, gastrointestinal (GI) toxicity and erectile dysfunction (ED), while intraoperative and postoperative day-30 morbidity consisted of the operation time, intraoperative blood loss, rate of conversion to open surgery, fibrosis in the radiotherapy area, etc.

Secondary endpoints The secondary endpoints compromised of the positive rate of post-operation incisal edge, biochemical progression-free survival (bPFS), postoperative continence and recovery of sexual function, quality of life (QoL), overall survival (OS) and adverse reactions of ADT.

Inclusion criteria

-Age 18-75 years at the time of registration

-Histologically confirmed adenocarcinoma of the prostate without small cell features

- Oligometastatic prostate cancer assessed by emission computed tomography (ECT), magnetic resonance imaging (MRI), Ga-68 Prostate-Specific Membrane Antigen(PSMA) PET/CT, Ga-68 PSMA $\mathrm{PET} / \mathrm{MR}$

$-<5$ oligometastases (including bone, lymph nodes above renal artery level, distant organ metastasis), and/or lymph nodes below renal artery level metastasis, tumor clinical stage cTx, Nx according to AJCC TNM 2017

-Expected survival time $>5$ years

-World Health Organization (WHO) performance status 0-1

-Written informed consent according to International Council for Harmonization/ Good Clinical Practice (ICH/GCP) regulations before registration and prior to any trial specific procedures

\section{Exclusion criteria}

-Any previous or ongoing treatment of prostate cancer including radiotherapy, ADT, chemotherapy, focal treatment, etc.

-Patients who have previously undergone transurethral prostatectomy or excision

-Patients who underwent other abdominal surgery within last 3 months

-Patients who had undergone transrectal prostatic biopsy within last 2 weeks 
-Patients with history of long-term use of anticoagulant and anti-platelet aggregation drugs and stopped anticoagulant less than 1 week before registration

-Patients with other malignancies, acute or chronic infections such as human immunodeficiency virus $(\mathrm{HIV})(+)$, hepatitis C virus (HCV) $(+)$ and / or positive syphilis

-Severe or active co-morbidity that is likely to impact the advisability of radiotherapy

-Any other serious underlying medical, psychiatric, psychological, familial, or geographical condition, which according to the judgement of the investigator may affect the planned staging, treatment and follow-up, patient compliance, or may cause high risk treatment-related complications for the patient -Patients who have participated in other clinical trials within last three months

-Patients who have refused to undergo RALP

-Unsuitable to participate in this clinical trial as per the judgement of the investigator

\section{Intervention}

In this trial, patients with oligometastatic prostate cancer are registered to receive the following treatment: $A D T$, radiotherapy and RALP. The trial schedule is illustrated in Fig. 1. Patients with oligometastatic prostate cancer receive 1 month of neoadjuvant ADT ( $\mathrm{nADT}$ ) followed by metastasis- directed radiation and abdominal or pelvic radiotherapy. Then offer RALP at the interval of 4-8 weeks after radiation, and ajuvant ADT for 2 years.

\section{ADT}

Both neoadjuvant and adjuvant ADT comprised of an oral antiandrogen (bicalutamide $50 \mathrm{mg}$ once daily) plus goserelin given subcutaneously 14 days after the initiation of bicalutamide as either $10.8 \mathrm{mg}$ with a second shot 3 months later or $3.6 \mathrm{mg}$ with a second shot 1 month later.

\section{Radiation therapy}

\subsection{Radiotherapy for oligometastases}

For oligometastases, the method and timing of radiotherapy varies depending on the location of metastasis.

For pelvic oligometastatic lesions, IMRT is administered simultaneously with prostate radiotherapy. The gross tumor volume (GTV1) of oligometastases is based on imaging examination: 68-Ga PSMA PET/CT, CT, MRI, ECT, etc. Planning target volumes (PTVs) for GTV1 are delineated with an additional $5 \mathrm{~mm}$ margin. Dose segmentation varies depending on the surrounding organs at risk (OARs) and tumor size. Firstly 49.5 Gy with 22 fractions or 50 Gy with 25 fractions is the recommended dose segmentation.

For non-pelvic oligometastatic lesions, SBRT will be administered. The gross tumor volume (GTV1) of oligometastases relied on imaging examination. PTV-GTV1: GTV1 relied on 3-5 mm uniform expansion. 6-8Gy per fraction with 5 fractions is the recommended dose segmentation which depends on the surrounding OARs and tumor size. If the oligometastases cause obvious symptoms, then SBRT is 
considered before prostate radiotherapy. If the oligometastases do not cause obvious symptoms, then prostate radiotherapy comes before SBRT. Dose guidelines to OARs in SBRT treatment is based upon AAPM Task Group 101 [17].

\subsection{Radiotherapy for prostate gland and pelvic or retroperitoneal metastatic lymph node (GTVnd)}

After the IMRT is administered, all patients have to undergo a contrasted CT simulation of the pelvis or abdomen of 5-mm-slice thickness in a supine position. Then, CT images will be transferred to the treatment planning system for contouring the target volume and OARs. Critical normal structures encompass the small bowel, bladder, femoral head, rectum, spinal cord, prostatic urethra (if visualised), bulb of urethra, kidney, etc. OARs should be contoured according to the pelvic normal tissue contouring guidelines of Radiation Therapy Oncology Group (RTOG) [18]. This protocol offers dose guidelines to OARs based on previous published RTOG trials [19].

The gross tumor volume (GTV2) is contoured based on the MRI. GTV2 consist of the prostate and seminal vesicle glands. GTVnd is further confirmed by medical imaging, whether CT, MRI, 68-Ga PSMA PET/CT, etc. The clinical tumor volume (CTV) compromises of a $5 \mathrm{~mm}$ margin to the GTV2, GTVnd, pelvic/retroperitoneal lymphatic drainage area.

The superior border of the whole pelvis field extends to the L5-S1 interspace for N1 subgroup. The pelvic lymphatic drainage area includes bilateral iliac lymph nodes, external iliac lymph nodes, internal iliac lymph nodes, S1-S3 levels presacral lymph nodes and obturator lymph nodes. The superior border of the retroperitoneal area is $2-3 \mathrm{~cm}$ above the positive lymph nodes though not crossing the renal artery level. PTV-GTV2: GTV2 is based on a 5-10 mm uniform expansion, but only $5 \mathrm{~mm}$ for the posterior part to reduce any rectal irradiation. PTV-GTVnd for GTVnd should be delineated with an additional $5 \mathrm{~mm}$ margin and PTV-CTV for CTV should be also delineated with an additional $5 \mathrm{~mm}$ margin separately.

Four radiation dose levels are planned as dose-escalation: $39.6 \mathrm{~Gy}, 45 \mathrm{~Gy}, 50.4 \mathrm{~Gy}$, and $54 \mathrm{~Gy}$ in 22 fractions, 25 fractions, 28 fractions, 30 fractions, respectively. Radiation therapy is delivered in 5 fractions per week. The initial two dose levels target whole pelvis/retroperitoneum, whereas the latter two dose levels act as a subsequent boost to the prostate, seminal vesicles and pelvic/retroperitoneal metastatic lymph nodes which will be added after reaching $45 \mathrm{~Gy}$.

\section{Dose escalation}

Dose escalation is conducted in a $3+3$ design with dose levels of $39.6,45,50.4$, and 54 Gy in 22, 25, 28 and 30 fractions respectively. A traditional $3+3$ dose-escalation design is adopted (Fig. 2). Briefly, three participants are initially allocated the starting dose cohort. If no dose-limiting toxicity (DLT) is observed in any of the three participants, the dose can be escalated and the three new patients can be enrolled to receive the next level of radiation dose. Even if one participant develops DLT, then additional three participants will be allocated the same dose cohort. If there are multiple observations of DLT at any given 
dose level, the dose escalation will be stopped and the previous dose level will be identified as the maximal tolerable dose (MTD). In this trial, DLTs are defined as any Grade III/IV toxicities.

\section{Radical prostatectomy}

Surgery can be scheduled 4-8 weeks after the completion of radiation therapy, via a robot-assisted laparoscopic approach and extended pelvic/retroperitoneal lymph node dissection should be performed.

\section{Follow-up}

68-Ga PSMA PET/CT should be conducted before registration, radiotherapy, surgery, and 1 year after the surgery. The prostate specific antigen (PSA) level of the participants should be check monthly. After the completion of treatment during the study, participants will be given a follow up every 3 months or as needed clinically in the first 2 years, every 6 months in the next 3 years, and annually thereafter. Follow-up examinations include clinical assessment, contrast-enhanced MRI, CT, ECT or 68-Ga PSMA PET/CT. For tumour progression, treatment alternatives can be evaluated inter-disciplinarily considering abiraterone acetate, enzalutamide, reradiation therapy, chemotherapy or others.

\section{Statistical analysis}

Normally distributed continuous data is described by means of \pm SD and $95 \%$ confidence intervals. Nonnormal distributional continuous data is described by the median and range. Qualitative ones is described by percentage. bPFS and OS are estimated using the Kaplan-Meier method. Univariate and multivariable hazard ratios are calculated using the Cox proportion hazard model. For comparisons between the baseline variables, the $\chi 2$ test and Fisher's exact test were performed. $P$ values $<0.05$ are considered statistically significant. Statistical analyses can be performed using SPSS software v18.0 or higher.

\section{Discussion}

For patients with oligometastatic prostate cancer, multimodality approach including consolidate local treatment, metastasis-directed therapy and systemic hormonal therapy might be the best to minimize the risk of recurrence.

Some other metastatic cancers have shown improved clinical outcomes with local aggressive approach for primary tumor and the cytoreductive radical prostatectomy may derive similar benefits in oligometastatic PCa by delaying the aggressive tumor burden [20-23]. Several retrospective studies have found that the safety and efficacy of radical prostatectomy in limited metastatic PCa with complication rates is comparable to locally advanced Pca [9, 24-27]. The first multicenter retrospective study include106 patients with M1a-b PCa reported perioperative complication (all Clavien 1-3) rates of 29\% [9]. Gandaglia et al. reported 7-year clinical progression-free rates of $45 \%$ and cancer-specific mortalityfree survival rates of $82 \%$ in a cohort of 11 patients with oligometastatic Pca [25]. Studies have shown the advantages of pre-operative radiotherapy over post-operative radiation in other malignancies $[28,29]$. 
Neoadjuvant radiotherapy, to some extent, could increase down-staging before radical prostatectomy and decrease the complexity of the surgery.

Furthermore, increasingly large number of data has shown that adoption of SBRT as metastasis-directed therapy for metastatic lesions of oligometastatic PCa provides excellent local control with minimal toxicity [30-33]. All of these studies reported zero ( $0 \%$ Grade 3 toxicities using CTCAE 5.0 criteria of adverse effects and $>95.5 \%$ local control rates.

Based on these promising results, our trial may represent an optimal treatment modality for oligometastatic prostate cancer. To acknowledge, our study is the first prospective trail to assess the safety and feasibility of metastasis-directed radiation and neoadjuvant hormone and radiation therapy followed by radical prostatectomy for oligometastatic PCa. Our project is expected to maximize the total tumor control outcomes while minimizing perioperative as well as long-term complications.

\section{Conclusion}

Metastasis-directed radiation and neoadjuvant hormone and radiation therapy followed by radical prostatectomy for treating oligometastatic prostate cancer is worth exploring.

\section{Abbreviations}

SBRT: Stereotactic body radiotherapy; ADT: Androgen deprivation therapy; bPFS: Biochemical progression-free survival; QoL:Quality of life; OS: Overall survival; PCa: Prostate cancer; RALP: Roboticassisted laparoscopic radical prostatectomy; IMRT: Intensity modulated radiation therapy; GU: Genitourinary; Gl: Gastrointestinal; ED: Erectile dysfunction; ECT: Emission computed tomography; MRI: Magnetic resonance imaging; PSMA: Prostate-Specific Membrane Antigen; WHO: World Health Organization; ICH/GCP: International Council for Harmonization/ Good Clinical Practice; GTV: Gross tumor volume; PTV:Planning target volume; OARs $₫$ Organs at risk; GTVnd: Pelvic or retroperitoneal metastatic lymph node; RTOG: Radiation Therapy Oncology Group; DLT: Dose-limiting toxicity; MTD: Maximal tolerable dose; PSA: Prostate specific antigen; RT: Radiation therapy; RP: Radical prostatectomy; Pt: Patient; Pts: Patients.

\section{Declarations}

Acknowledgements

None.

Funding

This study is sponsored by National Key Research and Development Program of China (2017YFC0113104), China Health Promotion Foundation THC2015001 and First Affiliated Hospital of 
Navy Medical University "234 Subject Climbing Program”.

Availability of data and materials

Materials and methods are available in the ChiCTR.

Authors' contributions

Study conception: H.J.Z, S.C.R Initial Study design: X.Z.Z., Y.T.X. and Y.F.C. Revision of study design and protocol: H.J.Z, S.C.R, X.Z.Z., Y.T.X. and Y.F.C. Study coordination: X.Z.Z., Y.T.X., Y.S.Y. Y.F.C. and L.G.J. Drafting the manuscript: X.Z.Z., Y.Y., and Y.T.X. Professionally stylistic ameliorations of the English language: M.E. All authors read and approved the final manuscript.

Ethics approval and consent to participate

Signature of the informed consent will be obtained from all patients before inclusion in the study. This study was approved by the Ethics committee of the Shanghai Changhai Hospital (CHEC2019-110) and is registered on ChiCTR (CHiCTR1900025743).

Consent for publication

Not applicable.

Competing interests

The authors declare that they have no competing interests.

\section{References}

1. [https://gco.iarc.fr/]

2. Mottet N, Bellmunt J, Bolla M, Briers E, Cumberbatch MG, De Santis M, Fossati N, Gross T, Henry AM, Joniau S, Lam TB, Mason MD, Matveev VB, Moldovan PC, Bergh RCN, Broeck TV, Poel HG, Kwast TH, Rouvière 0 , Schoots IG, Wiegel T, Cornford P. EAU-ESTRO-SIOG Guidelines on Prostate Cancer. Part 1: Screening, Diagnosis, and Local Treatment with Curative Intent. European urology. 2017;71(4):618629.

3. Cornford P, Bellmunt J, Bolla M, Briers E, De Santis M, Gross T, Henry AM, Joniau S, Lam TB, Mason MD, Poel HG, Kwast TH, Rouvière O, Wiegel T, Mottet N. EAU-ESTRO-SIOG Guidelines on Prostate Cancer. Part II: Treatment of Relapsing, Metastatic, and Castration-Resistant Prostate Cancer. European urology. 2017;71(4):630-642.

4. James ND, Spears MR, Clarke NW, Dearnaley DP, Mason MD, Parker CC, Ritchie AWS, Russell JM, Schiavone F, Attard G, Bono JS, Birtle A, Engeler DS, Elliott T, Matheson D, O'Sullivan J, Pudney D, Srihari N, Wallace J, Barber J, Syndikus I, Parmar MKB, Sydes MR. Failure-Free Survival and 
Radiotherapy in Patients With Newly Diagnosed Nonmetastatic Prostate Cancer: Data From Patients in the Control Arm of the STAMPEDE Trial. JAMA Oncol. 2016;2(3):348-357.

5. Lin CC, Gray PJ, Jemal A, Efstathiou JA. Androgen deprivation with or without radiation therapy for clinically node-positive prostate cancer. J Natl Cancer Inst. 2015;107(7):djv119.

6. Tward JD, Kokeny KE, Shrieve DC. Radiation therapy for clinically node-positive prostate adenocarcinoma is correlated with improved overall and prostate cancer-specific survival. Pract Radiat Oncol. 2013;3(3):234-240.

7. Rusthoven CG, Carlson JA, Waxweiler TV, Raben D, Dewitt PE, Crawford ED, Maroni PD, Kavanagh BD. The impact of definitive local therapy for lymph node-positive prostate cancer: a population-based study. Int J Radiat Oncol Biol Phys. 2014;88(5):1064-1073.

8. Aoun F, Peltier A, van Velthoven R. A comprehensive review of contemporary role of local treatment of the primary tumor and/or the metastases in metastatic prostate cancer. Biomed Res Int. 2014;2014:501213.

9. Sooriakumaran P, Karnes J, Stief C, Copsey B, Montorsi F, Hammerer P, Beyer B, Moschini M, Gratzke C, Steuber T, Suardi N, Briganti A, Manka L, Nyberg T, Dutton SJ, Wiklund P, Graefen M. A Multiinstitutional Analysis of Perioperative Outcomes in 106 Men Who Underwent Radical Prostatectomy for Distant Metastatic Prostate Cancer at Presentation. European urology. 2016;69(5):788-794.

10. Bayne CE, Williams SB, Cooperberg MR, Gleave ME, Graefen M, Montorsi F, Novara G, Smaldone MC, Sooriakumaran P, Wiklund PN, Chapin BF. Treatment of the Primary Tumor in Metastatic Prostate Cancer: Current Concepts and Future Perspectives. European urology. 2016;69(5):775-787.

11. Thompson IM, Tangen CM, Paradelo J, Lucia MS, Miller G, Troyer D, Messing E, Forman J, Chin J, Swanson G, Canby-Hagino E, Crawford ED. Adjuvant Radiotherapy for Pathological T3NOMO Prostate Cancer Significantly Reduces Risk of Metastases and Improves Survival: Long-Term Followup of a Randomized Clinical Trial. The Journal of urology. 2009;181(3):956-962.

12. Bolla M, van Poppel H, Tombal B, Vekemans K, Da Pozzo L, de Reijke TM, Verbaeys A, Bosset J-F, van Velthoven R, Colombel M, Beek C, Verhagen P, Bergh A, Sternberg C, Gasser T, Tienhoven G, Scalliet P, Haustermans K, Collette L. Postoperative radiotherapy after radical prostatectomy for high-risk prostate cancer: long-term results of a randomised controlled trial (EORTC trial 22911). The Lancet. 2012;380(9858):2018-2027.

13. Wiegel T, Bartkowiak D, Bottke D, Bronner C, Steiner U, Siegmann A, Golz R, Störkel S, Willich N, Semjonow A, Stöckle M, Rübe C, Rebmann U, Kälble T, Feldmann HJ, Wirth M, Hofmann R, EngenhartCabillic R, Hinke A, Hinkelbein W, Miller K. Adjuvant Radiotherapy Versus Wait-and-See After Radical Prostatectomy: 10-year Follow-up of the ARO 96-02/AUO AP 09/95 Trial. European urology. 2014;66(2):243-250.

14. van Gijn W, Marijnen CAM, Nagtegaal ID, Kranenbarg EM-K, Putter H, Wiggers T, Rutten HJT, PåhIman L, Glimelius B, van de Velde CJH. Preoperative radiotherapy combined with total mesorectal excision for resectable rectal cancer: 12-year follow-up of the multicentre, randomised controlled TME trial. The Lancet Oncology. 2011;12(6):575-582. 
15. O'Sullivan B, Davis AM, Turcotte R, Bell R, Catton C, Chabot P, Wunder J, Kandel R, Goddard K, Sadura A, Pater J, Zee B. Preoperative versus postoperative radiotherapy in soft-tissue sarcoma of the limbs: a randomised trial. The Lancet. 2002; 359(9325):2235-2241.

16. Supiot S, Shubbar S, Fleshner N, Warde P, Hersey K, Wallace K, Cole H, Sweet J, Tsihlias J, Jewett MAS, Klotz L, Bristow RG. A phase I trial of pre-operative radiotherapy for prostate cancer: Clinical and translational studies. Radiotherapy and Oncology. 2008;88(1):53-60.

17. Benedict SH, Yenice KM, Followill D, Galvin JM, Hinson W, Kavanagh B, Keall P, Lovelock M, Meeks S, Papiez L, Purdie T, Sadagopan R, Schell MC, Salter B, Schlesinger DJ, Shiu AS, Solberg T, Song DY, Stieber V, Timmerman R, Tomé WA, Verellen D, Wang L, Yin FF. Stereotactic body radiation therapy: the report of AAPM Task Group 101. Med Phys. 2010;37(8):4078-101.

18. Gay HA, Barthold HJ, O'Meara E, Bosch wr, Naqa IE, Al-Lozi R, Rosenthal SA, Lawton C, Lee WR, Sandler H, Zietman A, Myerson R, Dawson LA, Willett C, Kachnic LA, Jhingran A, Portelance L, Ryu J, Jr WS, Gaffney D, Viswanathan AN, Michalski JM. Pelvic normal tissue contouring guidelines for radiation therapy: a Radiation Therapy Oncology Group consensus panel atlas. Int J Radiat Oncol Biol Phys. 2012;83(3):e353-62.

19. Lawton CA囚Miehalski J囚EI-Naqa I囚 Buyyounouski MK, Lee WR, Menard C, O'Meara E, Rosenthal SA, Ritter M, Seider M. RTOG GU Radiation oncology specialists reach consensus on pelvic lymph node volumes for high-risk prostate cancer. Int J Radiat Oncol Biol Phys. 2009;74(2):383-387】

20. Mickisch GH, Garin A, van Poppel H, de Prijck L, Sylvester R. European Organisation for R, Treatment of Cancer Genitourinary G: Radical nephrectomy plus interferon-alfa-based immunotherapy compared with interferon alfa alone in metastatic renal-cell carcinoma: a randomised trial. Lancet (London, England). 2001;358(9286):966-970.

21. Flanigan RC, Mickisch G, Sylvester R, Tangen C, Van Poppel H, Crawford ED. Cytoreductive nephrectomy in patients with metastatic renal cancer: a combined analysis. The Journal of urology. 2004;171(3):1071-1076.

22. Bristow RE, Tomacruz RS, Armstrong DK, Trimble EL, Montz FJ. Survival effect of maximal cytoreductive surgery for advanced ovarian carcinoma during the platinum era: a meta-analysis. $J$ Clin Oncol. 2002;20(5):1248-1259.

23. Glehen O, Mohamed F, Gilly FN. Peritoneal carcinomatosis from digestive tract cancer: new management by cytoreductive surgery and intraperitoneal chemohyperthermia. Lancet Oncol. 2004;5(4):219-228.

24. Heidenreich A, Pfister D, Porres D. Cytoreductive radical prostatectomy in patients with prostate cancer and low volume skeletal metastases: results of a feasibility and case-control study. The Journal of urology. 2015; 193(3):832-838.

25. Gandaglia G, Fossati N, Stabile A, Bandini M, Rigatti P, Montorsi F, Briganti A. Radical Prostatectomy in Men with Oligometastatic Prostate Cancer: Results of a Single-institution Series with Long-term Follow-up. European urology. 2017;72(2):289-292. 
26. Ward JF, Slezak JM, Blute ML, Bergstralh EJ, Zincke H. Radical prostatectomy for clinically advanced (cT3) prostate cancer since the advent of prostate-specific antigen testing: 15-year outcome. BJU international. 2005;95(6):751-756.

27. Loeb S, Smith ND, RoehI KA, Catalona WJ. Intermediate-term potency, continence, and survival outcomes of radical prostatectomy for clinically high-risk or locally advanced prostate cancer. Urology. 2007;69(6):1170-1175.

28. Sauer R, Liersch T, Merkel S, Fietkau R, Hohenberger W, Hess C, Becker H, Raab H-R, Villanueva M-T, Witzigmann $\mathrm{H}$, Wittekind C, Beissbarth T, Rödel C. Preoperative versus postoperative chemoradiotherapy for locally advanced rectal cancer: results of the German CAO/ARO/AIO-94 randomized phase III trial after a median follow-up of 11 years. J Clin Oncol. 2012;30(16):1926-1933.

29. Roh MS, Colangelo LH, O'Connell MJ, Yothers G, Deutsch M, Allegra CJ, Kahlenberg MS, Baez-Diaz L, Ursiny CS, Petrelli NJ, Wolmark N. Preoperative multimodality therapy improves disease-free survival in patients with carcinoma of the rectum: NSABP R-03. J Clin Oncol. 2009;27(31):5124-5130.

30. Ahmed KA, Barney BM, Davis BJ, Park SS, Kwon ED, Olivier KR. Stereotactic body radiation therapy in the treatment of oligometastatic prostate cancer. Front Oncol. 2013;2:215.

31. Berkovic P, De Meerleer G, Delrue L, Lambert B, Fonteyne V, Lumen N, Decaestecker K, Villeirs G, Vuye $\mathrm{P}$, Ost P. Salvage stereotactic body radiotherapy for patients with limited prostate cancer metastases: deferring androgen deprivation therapy. Clin Genitourin Cancer. 2013;11(1):27-32.

32. Schick U, Jorcano S, Nouet P, Rouzaud M, Vees H, Zilli T, Ratib O, Weber DC, Miralbell R. Androgen deprivation and high-dose radiotherapy for oligometastatic prostate cancer patients with less than five regional and/or distant metastases. Acta Oncol. 2013;52(8):1622-1628.

33. Decaestecker K, De Meerleer G, Lambert B, Delrue L, Fonteyne V, Claeys T, De Vos F, Huysse W, Hautekiet A, Maes G, Ost P. Repeated stereotactic body radiotherapy for oligometastatic prostate cancer recurrence. Radiat Oncol. 2014; 9:135.

\section{Figures}




\begin{tabular}{|c|c|c|c|c|c|c|}
\hline Time Course & $1 \mathrm{M}$ & $1-2 \mathrm{~W}$ & $5-8 \mathrm{~W}$ & $4-8 \mathrm{~W}$ & $1 \mathrm{D}$ & $2 \mathrm{Y}$ \\
\hline ADT & & & & & & \\
\hline RT & & RT for O & RT for P & & & \\
\hline RP & & & & & & \\
\hline
\end{tabular}

\section{Figure 1}

ADT: androgen deprivation therapy

$\mathrm{RT}$ : radiation therapy

$\mathrm{RP}$ : radical prostatectomy

Treatment schedule in the protocol. M: month; W: week; D: day; Y: year.

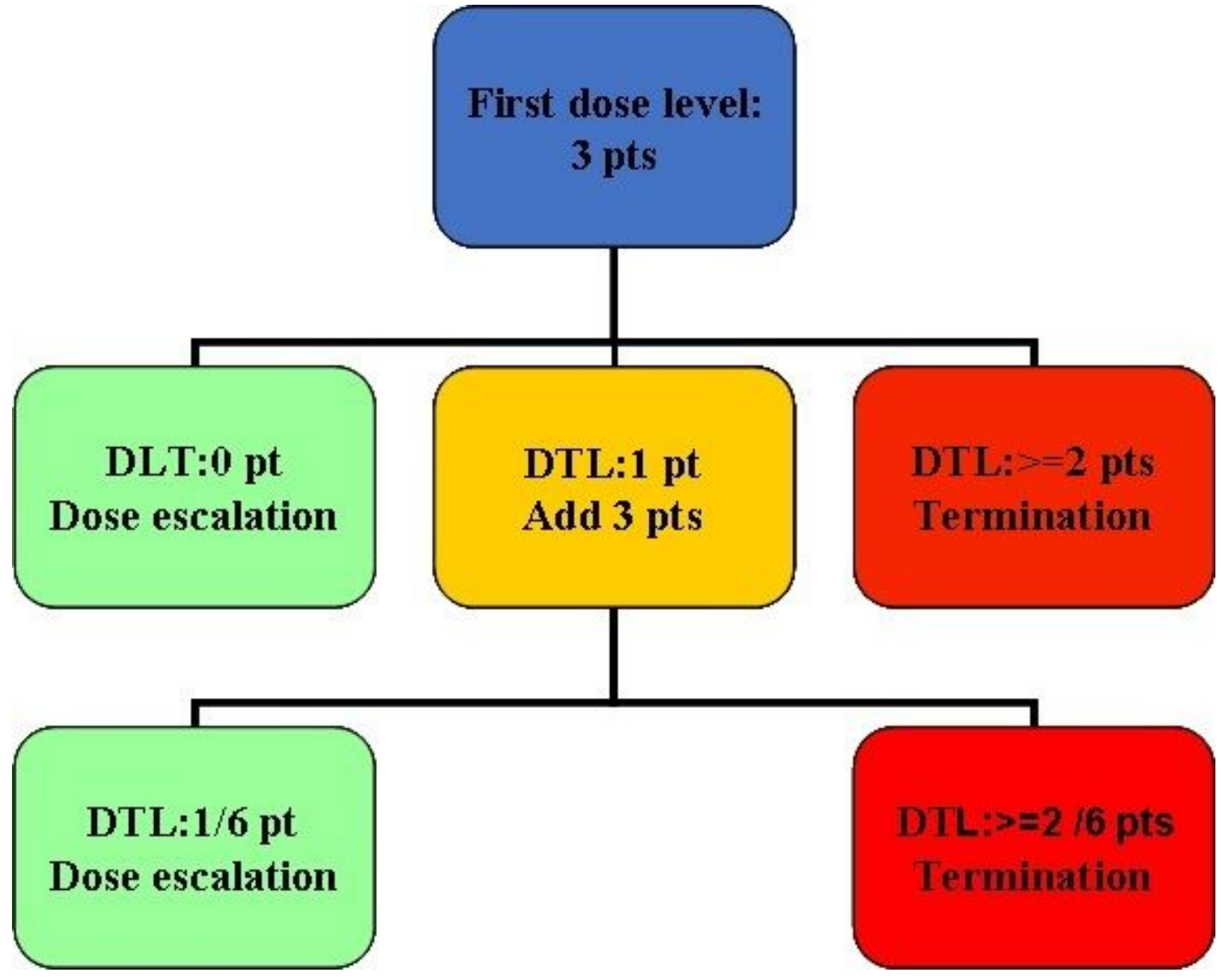

Figure 2 
Graphical depiction of traditional 3+3dose escalation methods for the clinical trials. DLT, dose limiting toxicity; MTD, maximum tolerance dose; Pt, patient; Pts, patients. 